\title{
Response to an external magnetic field of the decay rate of a neutral scalar field into a charged fermion pair
}

\author{
Jorge Jaber-Urquiza, ${ }^{1, *}$ Gabriella Piccinelli, ${ }^{2, \dagger}$ and Angel Sánchez ${ }^{1, \$}$ \\ ${ }^{1}$ Facultad de Ciencias, Universidad Nacional Autónoma de México, \\ Apartado Postal 50-542, Ciudad de México 04510, Mexico \\ ${ }^{2}$ Centro Tecnológico, Facultad de Estudios Superiores Aragón, Universidad Nacional Autónoma de México, \\ Avenida Rancho Seco S/N, Col. Impulsora Popular Avícola, \\ Nezahualcóyotl, Estado de México 57130, Mexico
}

(Received 19 October 2018; revised manuscript received 15 February 2019; published 22 March 2019)

In this work, we explore the effects of a weak magnetic field on the decay process of a neutral scalar boson into a pair of charged fermions in vacuum. Since the analytical computation of the decay width needs some approximation, following two different approaches, we study the low and high transverse momentum limits. Our findings indicate that the magnetic field effect depends on the kinematics of the scalar particle.

DOI: 10.1103/PhysRevD.99.056011

\section{INTRODUCTION}

Scalar fields appear in several branches of physics, in different ways, as fundamental or composite fields. At different energy scales, they can be present in condensed matter (superconductors [1]), compact astrophysical objects (color superconductivity [2] and superfluidity [3]), high energy physics (Higgs physics and heavy ion collisions [4-6]), and cosmological events (where the scalar field can be a matter [7] and energy [8] component of the dark sector, or the inflaton; see e.g., [9]).

On the other hand, in all these processes, we can also expect the presence of a magnetic field. Thus, the following is a natural question that emerges: how does this magnetic field change the physics in the phenomena driven by the scalar field? Several authors have addressed this question, finding different answers and eventually discovering new phenomena, such as direct and inverse magnetic catalysis $[10,11]$. Some of these works have been developed in inflation [12], electroweak phase transition and baryogenesis [13], and QCD phase diagrams in high density [14] and temperature [15] regions (for recent reviews, see [16]).

Other answers have also come from the study of the effect of a magnetic field on the particle decay process. This effect has been studied in different situations: high intensity

\footnotetext{
*jorgejaber@ciencias.unam.mx

itzamna@unam.mx

\#ansac@ciencias.unam.mx
}

Published by the American Physical Society under the terms of the Creative Commons Attribution 4.0 International license. Further distribution of this work must maintain attribution to the author(s) and the published article's title, journal citation, and DOI. Funded by SCOAP ${ }^{3}$. laser experiments [17], heavy ion collisions [6,18,19], compact objets [20,21], and early universe events [22-24].

In all these contexts, the decay processes take place in external conditions with different temperature, density, electromagnetic field strength, and type of progenitor and decay products [25].

In the cases presented in the literature, two limits are typically considered: strong magnetic fields, in which case only the lowest Landau level (LLL) is taken into account, and weak magnetic fields, that allow us to perform some kind of expansion series in $B$ (with $B$ the magnetic field) and keep only the leading terms. In addition, the methods followed to calculate the decay rate and the approximations necessary to the calculations vary from one work to another. Even though all these differences are expected to have consequences for the process, it is nonetheless remarkable that different results can be found in the literature for the same physical process.

In addition to the first works on decay processes in the presence of an external magnetic field [26-32], there is once again intense activity around this subject $[18,19,24,33-39]$. It is nonetheless important to further explore this area in order to discern which are the relevant physical ingredients and to make the results converge when they describe the same physical situation. Moreover, it is crucial to delimit the magnetic field effect on the collected data in experiments since any deviation from the expected result could be misinterpreted as new physics.

In a recent publication [22], we have shown that the kinematics of the progenitor particle plays an important rôle in the decay process. This ingredient should be taken into account since, depending on the kinematic region of interest, the decay width can be enhanced or inhibited by the external magnetic field. In the present work, we extend 
these ideas by exploring another important aspect on the decay process: the spin.

In order to explore the effect of external magnetic fields on the decay process and its possible relation with spin, in this work we shall study a heavy scalar boson decay into two charged fermions, in vacuum, with different approaches. In particular, we are interested in the weak field limit, in such a way that our study could be applied to particle production during late stages of peripheral heavy ion collisions [40] and in the inflaton decay process in a warm inflation scenario [41-43], considering that cosmic magnetic fields observed at all scales in the Universe $[44,45]$ could be primordial [46-48].

The outline of this work is as follows: in Sec. II, we present the model and calculate the scalar self-energy in the presence of an external homogeneous magnetic field by considering the interaction of a neutral scalar with a pair of charged fermions, up to one loop; in Sec. III, we get the heavy boson self-energy in the weak field limit through two different approximations that take care of the kinematic along the transverse direction with respect to the magnetic field and obtain the decay width by invoking the optical theorem; results are presented in Sec. IV, together with a discussion on the physical differences between these two approaches. Finally, Sec. V contains our conclusions.

\section{MODEL}

Let us consider a model in which a neutral scalar boson $\phi$ interacts with two charged fermions $\bar{\psi} \psi$. The simplest form for a Lagrangian to account for this process is

$$
\mathcal{L}=g \phi \bar{\psi} \psi
$$

This interaction term gives rise to the Feynman diagram shown in Fig. 1, whose analytical expression reads

$$
\Sigma^{B}(x, y)=i g^{2} \operatorname{tr}\left[S_{F}^{B}(x, y) S_{F}^{B}(y, x)\right] .
$$

To consider the effect of an external magnetic field, we use the Schwinger's proper-time method [49], that incorporates the full magnetic contribution into the momentumdependent propagators. In such a way, the propagator has the form

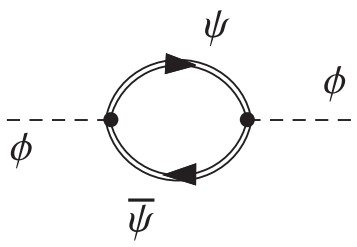

FIG. 1. Leading-order contribution to the neutral scalar $\phi$ boson self-energy in a magnetic field background. $\psi^{\prime} s$ are charged fermions and the double dashed lines represent their propagators dressed with the magnetic field.

$$
S_{F}^{B}(x, y)=\Omega(x, y) \int \frac{d^{4} k}{(2 \pi)^{4}} S_{F}^{B}(k) e^{-i k(x-y)}
$$

where

$$
\begin{aligned}
S_{F}^{B}(k)= & \int_{0}^{\infty} \frac{d s}{\cos (e B s)} e^{-i s\left(m^{2}-k_{\|}^{2}-k_{\perp}^{2} \frac{\tan (e B s)}{e B s}\right)} \\
& \times\left[\left(m+\not k_{\|}\right) e^{-i e B s \Sigma_{3}}+\frac{\not k_{\perp}}{\cos (e B s)}\right],
\end{aligned}
$$

and

$$
\Omega\left(x^{\prime}, x^{\prime \prime}\right)=\exp \left(-i e \int_{x^{\prime \prime}}^{x^{\prime}} A_{\mu}(x) d x^{\mu}\right)
$$

is the well-known Schwinger's phase which encodes the gauge dependence of the external magnetic field, and $e$ and $m$ denote the charge and mass of the fermion field $\psi$, respectively; $B$ is the external magnetic field, and $\Sigma_{3}=i \gamma_{1} \gamma_{2}$, with $\gamma$ 's the Dirac gamma matrices. Notice that this particular form for the propagator implies that we have considered an external uniform magnetic field along the $z$ direction, allowing us to adopt the notation $a \cdot b \equiv(a \cdot b)_{\|}+(a \cdot b)_{\perp}$, with $\quad(a \cdot b)_{\|} \equiv a^{0} b^{0}-a^{3} b^{3}$ and $(a \cdot b)_{\perp} \equiv-a^{1} b^{1}-a^{2} b^{2}$.

In the momentum space, the self-energy reads

$$
\Sigma(p)=i g^{2} \int \frac{d^{4} k}{(2 \pi)^{4}} \operatorname{tr}\left[S_{F}^{B}(k) S_{F}^{B}(k-p)\right]
$$

where $p$ and $k$ denote the decaying and product particles momenta, respectively. Note that the phase has canceled identically, due to a close loop of charged fermions. Once we replace the propagators from Eq. (4) in Eq. (6), the scalar self-energy dressed with a magnetic field reads

$$
\begin{aligned}
& \Sigma^{B}(p)=i g^{2} \int \frac{d^{4} k}{(2 \pi)^{4}} \int_{0}^{\infty} d s_{1} \int_{0}^{\infty} d s_{2} \frac{e^{-i m^{2}\left(s_{1}+s_{2}\right)}}{\cos \left(e B s_{1}\right) \cos \left(e B s_{2}\right)} \\
& \times e^{i s_{1}\left(k_{\|}^{2}+k_{\perp}^{2} \frac{\tan \left(e B s_{1}\right)}{e B s_{1}}\right)} e^{i s_{2}\left((k-p)_{\|}^{2}+(k-p)_{\perp}^{2} \frac{\tan \left(e B s_{2}\right)}{e B s_{2}}\right)} \\
& \times \operatorname{tr}\left\{\left[\left(m+\not \|_{\|}\right) e^{-i e B s_{1} \Sigma_{3}}+\frac{\not K_{\perp}}{\cos \left(e B s_{1}\right)}\right]\right. \\
& \left.\times\left[\left(m+\not \ell_{\|}-\not p_{\|}\right) e^{-i e B s_{2} \Sigma_{3}}+\frac{\not \not_{\perp}-\not p_{\perp}}{\cos \left(e B s_{2}\right)}\right]\right\} \text {. }
\end{aligned}
$$

Now, by using the change of variables,

$$
s=s_{1}+s_{2} \quad \text { and } \quad v=\frac{s_{1}-s_{2}}{s_{1}+s_{2}},
$$

and performing the trace over Dirac's gamma matrices, as well as the Gaussian integrals over the momenta $k$, we finally arrive at 


$$
\begin{aligned}
\Sigma^{B}(p)= & \frac{g^{2} e B}{8 \pi^{2}} \int_{0}^{\infty} d s \int_{-1}^{1} d v e^{-i s m^{2}} e^{i \frac{i\left(1-v^{2}\right)}{4} p_{\|}^{2}} e^{\frac{i \cos (e B s v)-\cos (e B s)}{e B(\sin (e B s)}} p_{\perp}^{2} \\
& \times\left[\frac{1}{\tan (e B s)}\left(m^{2}+\frac{i}{s}-\frac{1-v^{2}}{4} p_{\|}^{2}\right)+\frac{i e B}{\sin ^{2}(e B s)}\right. \\
& \left.-\frac{\cos (e B s v)-\cos (e B s)}{2 \sin ^{3}(e B s)} p_{\perp}^{2}\right]
\end{aligned}
$$

which accounts for the effect of a magnetic field on the scalar self-energy due to a loop of charged fermions. Equation (9) is an exact result, since we have not made any approximation on the magnetic field strength. However, the remaining integrals cannot be calculated analytically because of the involved form of the self-energy. To gain some insight about the magnetic field effect on the analytical structure of the self-energy, in what follows we shall explore two approximations.

\section{MAGNETIC FIELD EFFECT ON SCALAR DECAY INTO FERMIONS}

Taking into account that in Eq. (9) the physical scales are the progenitor particle momentum $p$, the daughter particle mass $m$ and the magnetic strength interaction $e B$, the approximations that can be done depend on the hierarchy of scales among these quantities. In particular, as we mentioned in the Introduction, we are interested in weak magnetic fields with respect to the mass $m$, i.e., $e B \ll m^{2}$. In this regime, we still have two possibilities depending on the third physical scale involved in the selfenergy, the transverse momentum. As can be seen in Eq. (9), there is an exponential factor that involves the combination of the transverse momentum and the magnetic field. In this way, we should be careful with the expansion of this term. In what follows, we study two different approximations depending on the progenitor particle kinematics.

\section{A. Weak magnetic field and low transverse momentum limit}

Let us start by considering the limit in which both the magnetic field and the decaying particle transverse momentum are low and of the same order of magnitude. In this case, all the factors in the integrand of Eq. (9) can be expanded up to $(e B)^{2}$ terms and the self-energy can be written as

$$
\Sigma^{B}(p) \simeq \Sigma(0)+\tilde{\Sigma}(p)+\tilde{\Sigma}^{B}(p)
$$

where the first two terms correspond to the self-energy in vacuum and are given by

$$
\Sigma(0)=\frac{g^{2}}{4 \pi^{2}} \int_{0}^{\infty} \frac{d s}{s} e^{-i m^{2} s}\left[\frac{2 i}{s}+m^{2}\right]
$$

which is the contribution that contains the divergencies associated with the mass $m$ and the coupling constant $g$, and

$$
\begin{aligned}
\tilde{\Sigma}(p)= & \frac{g^{2}}{8 \pi^{2}}\left[\left(\frac{p^{4}}{2}-2 m^{2} p^{2}\right) \int_{-1}^{1} d v \frac{v^{2}}{\left(1-v^{2}\right) p^{2}-4 m^{2}}\right. \\
& \left.+\frac{p^{4}}{2} \int_{-1}^{1} d v \frac{v^{4}}{\left(1-v^{2}\right) p^{2}-4 m^{2}}-p^{2} \int_{0}^{\infty} \frac{d s}{s} e^{-i s m^{2}}\right],
\end{aligned}
$$

that is composed by two finite terms (the first two terms) and a divergent term (the last one) related to the wave function. Since these divergencies are related with physical scales, all of them are real quantities and will not affect the imaginary part, in which we are interested here.

Finally, the magnetic contribution in Eq. (10) is

$$
\begin{aligned}
\tilde{\Sigma}^{B}(p)= & \frac{g^{2}}{8 \pi^{2}}\left[\frac{16}{3}(e B)^{2} m^{2} \int_{-1}^{1} \frac{d v}{\left(\left(1-v^{2}\right) p^{2}-4 m^{2}\right)^{2}}-\frac{4}{3}(e B)^{2} p_{\|^{2}} \int_{-1}^{1} d v \frac{1-v^{2}}{\left(\left(1-v^{2}\right) p^{2}-4 m^{2}\right)^{2}}\right. \\
& +\frac{8}{3}(e B)^{2} p_{\perp}^{2} m^{2} \int_{-1}^{1} d v \frac{\left(1-v^{2}\right)^{2}}{\left(\left(1-v^{2}\right) p^{2}-4 m^{2}\right)^{3}}-\frac{2}{3}(e B)^{2} p^{2} p_{\perp}^{2} \int_{-1}^{1} d v \frac{\left(1-v^{2}\right)^{3}}{\left(\left(1-v^{2}\right) p^{2}-4 m^{2}\right)^{3}} \\
& \left.+\frac{1}{3}(e B)^{2} p_{\perp}^{2} \int_{-1}^{1} d v \frac{\left(1-v^{2}\right)(7-3 v)}{\left(\left(1-v^{2}\right) p^{2}-4 m^{2}\right)^{2}}\right] .
\end{aligned}
$$

Note that by doing this expansion we have taken into account that the main contribution to the integral comes from the region $e B s \ll 1$ [26,27]. In Eqs. (11), (12), and (13), the integrals over $v$ or $s$ have been performed when the operation became trivial.

In order to calculate the decaying rate, we invoke the optical theorem that relates the imaginary part of the selfenergy and the decay rate as [50]

$$
\Gamma=-\frac{\Im(\Sigma(p))}{\sqrt{\vec{p}^{2}+M^{2}}},
$$

where the imaginary part of the self-energy, up to one loop, is represented by cutting the Feynman diagram, as shown in Fig. 2. 


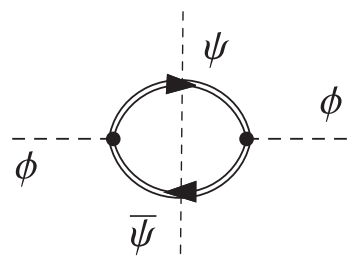

FIG. 2. Schematic representation of the imaginary part of the self-energy, obtained by cutting the one-loop Feynman diagram.

Replacing Eq. (10) into Eq. (14) and bearing in mind that the mass $m$ has an $i \epsilon$ term that warrants the causality in the propagator in Eq. (4), the calculation of the imaginary part can be easily done by using the identities $[51,52]$

$$
\lim _{\epsilon \rightarrow 0} \Im\left(\frac{1}{(x+i \epsilon)^{n}}\right)=\frac{(-1)^{n+1}}{n !} \frac{d^{n} \delta(x)}{d x^{n}},
$$

and

$$
\int_{-\infty}^{\infty} \frac{d^{m} \delta(x-a)}{d x^{m}} f(x) d x=\left.(-1)^{m} \frac{d^{m} f(x)}{d x^{m}}\right|_{x=a} .
$$

In such a way, we obtain for the decay rate

$$
\begin{aligned}
\Gamma^{B}= & \frac{2 g^{2}}{16 \pi}\left[\frac{\left(p^{2}-4 m^{2}\right)^{3 / 2}}{p}-\frac{24(e B)^{2} m^{4} p_{\perp}^{2}}{p^{5}\left(p^{2}-4 m^{2}\right)^{3 / 2}}\right. \\
& -\frac{16(e B)^{2} m^{2} p_{\perp}^{2}}{3 p^{5}} \frac{12 m^{4}-7 m^{2} p^{2}+p^{4}}{\left(p^{2}-4 m^{2}\right)^{5 / 2}} \\
& \left.+\frac{4(e B)^{2}}{3 p^{3}} \frac{4 m^{2} p^{2}+2 m^{2} p_{\perp}^{2}+2 p^{2} p_{\perp}^{2}-p^{4}}{\left(p^{2}-4 m^{2}\right)^{3 / 2}}\right] \\
& \times \frac{\Theta\left(p^{2}-4 m^{2}\right)}{\sqrt{\vec{p}^{2}+M^{2}}} .
\end{aligned}
$$

In Appendix, we show an alternative way to calculate the magnetic contribution in the above equation.

In particular, for an on-shell scalar particle in its rest frame $\left(p^{2}=M^{2}\right)$, the decay width reads

$$
\Gamma^{B}=\frac{2 g^{2}}{16 \pi}\left[\frac{\left(M^{2}-4 m^{2}\right)^{3 / 2}}{M^{2}}-\frac{4(e B)^{2}}{3 M^{2} \sqrt{M^{2}-4 m^{2}}}\right] \Theta\left(M^{2}-4 m^{2}\right) .
$$

The apparent divergencies near the threshold $p=2 \mathrm{~m}$ in the second term of this equation, as well as in several terms of Eq. (17), are in fact not present since, in order to be consistent with the weak field limit expansion, it is required that the magnetic field strength goes to zero faster than the terms that contain the threshold information. This statement comes from the fact that in the expansion shown in Eq. (17), the expansion parameter is $e B /\left(p^{2}-4 m^{2}\right)$. This, in turn, goes back to Eq. (9), which, in the absence of a magnetic field, has two different physical scales: $\mathrm{m}^{2}$ and $\left(p^{2}-4 m^{2}\right)$, which correspond to the extreme values in the argument of the exponential. The last one is the relevant scale for the development of the self-energy imaginary part (see also [24]).

\section{B. Weak magnetic field and high transverse momentum limit}

Let us now explore the other weak field limit: $e B \ll m^{2} \ll p_{\perp}^{2}$. In this case we must carry out a Taylor expansion with care [26]: the argument in the exponential that involves the magnetic field is expanded up to $(e B s)^{2}$, however, since there are terms that contain a factor $p_{\perp}^{2}$ that can be large (known as crossed field approximation), then, the exponential itself cannot be expanded in powers of $e B s$. Bearing this in mind, we get

$$
\begin{aligned}
\Sigma^{B}(p) \simeq & \frac{g^{2}}{8 \pi^{2}} \int_{0}^{\infty} d s \int_{-1}^{1} d v e^{-i s m^{2}} e^{i \frac{s\left(1-v^{2}\right)}{4} p^{2}} e^{i s^{\frac{3(e B)^{2}}{48}\left(1-v^{2}\right)^{2} p_{\perp}}} \\
& \times\left[\frac{m^{2}}{s}+\frac{2 i}{s^{2}}-\frac{1-v^{2}}{4 s} p^{2}-\frac{m^{2}(e B)^{2} s}{3}\right. \\
& +\frac{(e B)^{2} s}{12}\left(1-v^{2}\right) p_{\|}^{2} \\
& \left.-\frac{(e B)^{2} s}{48}\left(1-v^{2}\right)\left(5-v^{2}\right) p_{\perp}^{2}\right]
\end{aligned}
$$

In a similar way as we proceeded in the previous section, we can decompose the self-energy as

$$
\Sigma^{B}(p) \simeq \Sigma(0)+\tilde{\Sigma}^{B}(p),
$$

with $\Sigma(0)$ the same term as in Eq. (11), and

$$
\begin{aligned}
\tilde{\Sigma}^{B}(p)= & \frac{g^{2}}{8 \pi^{2}}\left[-p^{2} \int_{0}^{\infty} \frac{d s}{s} e^{-i s m^{2}}-\frac{(e B)^{2}}{3}\left(m^{2}-\frac{p_{\|}^{2}}{4}+\frac{5 p_{\perp}{ }^{2}}{16}\right) V_{0} S_{1}+\frac{i}{2}\left(m^{2} p^{2}-\frac{p^{4}}{4}\right) V_{2} S_{0}-\frac{(e B)^{2}}{12}\left(p_{\|}^{2}+\frac{p_{\perp}{ }^{2}}{2}\right) V_{2} S_{1}\right. \\
& \left.+i \frac{(e B)^{2}}{12} p_{\perp}^{2}\left(m^{2}-\frac{p^{2}}{4}\right) V_{2} S_{2}-i \frac{p^{4}}{8} V_{4} S_{0}+\frac{7(e B)^{2}}{48} p_{\perp}^{2} V_{4} S_{1}-i \frac{m^{2}(e B)^{2}}{12} p_{\perp}^{2} V_{4} S_{2}+i \frac{(e B)^{2}}{48} p^{2} p_{\perp}^{2} V_{6} S_{2}\right],
\end{aligned}
$$


where, for simplicity, we have introduced the notation

$$
V_{n} S_{m} \equiv \int_{-1}^{1} d v v^{n} \int_{0}^{\infty} d s s^{m} e^{i s\left(\frac{1-v^{2}}{4} p^{2}-m^{2}+s^{2} \frac{(e B)^{2}}{48}\left(1-v^{2}\right)^{2} p_{\perp}{ }^{2}\right)}
$$

It is worth to notice that in this case it was not possible to identify the $\tilde{\Sigma}(p)$, like in Eq. (10), due to the fact that the integrals include a new exponential factor.

Once we replace Eq. (20) in Eq. (14), we arrive at

$$
\begin{aligned}
\Gamma^{B}= & \frac{g^{2}}{16 \pi} \frac{8 \sqrt[3]{2}}{(e B)^{2 / 3} p_{\perp}^{2 / 3}}\left[-\frac{p^{2}}{2} \int_{\sqrt{1-\frac{4 m^{2}}{p^{2}}}}^{1} d v\left(1-v^{2}\right)^{-2 / 3} v^{2}\left(\frac{1+v^{2}}{4} p^{2}-m^{2}\right) A i(x)\right. \\
& +\frac{4}{3} \int_{\sqrt{1-\frac{4 m^{2}}{p^{2}}}}^{1} d v\left(1-v^{2}\right)^{-5 / 3} v^{2}\left(\frac{1+v^{2}}{4} p^{2}-m^{2}\right)\left(\frac{1-v^{2}}{4} p^{2}-m^{2}\right) A i(x) \\
& \left.+\frac{2 \sqrt[3]{2}(e B)^{4 / 3}}{3 p_{\perp}^{2 / 3}} \int_{\sqrt{1-\frac{4 m^{2}}{p^{2}}}}^{1} d v\left(1-v^{2}\right)^{-4 / 3}\left(\frac{1-v^{2}}{4} p_{\|}^{2}-\frac{\left(1-v^{2}\right)\left(7 v^{2}-5\right)}{16} p_{\perp}^{2}-m^{2}\right) A i^{\prime}(x)\right] \frac{\Theta\left(p^{2}-4 m^{2}\right)}{\sqrt{\vec{p}^{2}+M^{2}}}
\end{aligned}
$$

where the terms that involved the integration over $s$ have been identified with the integral representation of the first kind Airy functions

$$
\operatorname{Ai}(x)=\frac{1}{\pi} \int_{0}^{\infty} \cos \left(x t+\frac{t^{3}}{3}\right) d t
$$

and its derivatives [53], with

$$
x=\frac{m^{2}-\frac{1-v^{2}}{4} p^{2}}{\left(\frac{-p_{\perp}^{2}(e B)^{2}\left(1-v^{2}\right)^{2}}{16}\right)^{1 / 3}} .
$$

Note that the zero magnetic field limit can be recovered by using the Dirac delta representation [53]

$$
\delta(x) \equiv \lim _{\varepsilon \rightarrow 0} \frac{1}{\varepsilon} A i\left(\frac{x}{\varepsilon}\right) \quad \text { with } \quad \varepsilon \equiv\left(\frac{(e B)^{2}}{m^{4}}\right)^{\frac{1}{3}} .
$$

It is worth keeping in mind that this approximation does not allow us to study the decay process in the particle rest frame since, by construction, $p_{\perp}$ is large. In the next section, we present our results and discuss the rôle played by the decaying particle kinematics: $p_{\perp} \sim m$ and $p_{\perp} \gg m$.

\section{RESULTS AND DISCUSSION}

The decay rate as a function of the magnetic field normalized with the fermion mass $m$, in the regime of low transverse momentum $e B \sim p_{\perp}^{2} \leq m^{2}<p^{2}$, Eq. (17), presents a slight suppression as the magnetic field increases. In order to quantify the magnetic field effect on the decay process it is convenient to compare it with the decay rate in vacuum,

$$
\Delta \Gamma\left(p, p_{\perp}, m, e B\right) \equiv \frac{\Gamma^{B}\left(p, p_{\perp}, m, e B\right)}{\Gamma_{\mathrm{vac}}\left(p, p_{\perp}, m\right)},
$$

where $\Gamma_{\text {vac }}\left(p, p_{\perp}, m\right)$ corresponds to the first term in Eq. (17). For definiteness, we call this expression the decay response to the external magnetic field. In this way, we also eliminate the relativistic effect due to dilatation of time and the dependence on $e B$ can more easily be seen. To have an idea of the suppression strength, with the parameters $p / m=5$ and $p_{\perp} / m=0.1$ for $e B / m^{2}$ within $(0,0.2)$, the response of the decay to the external magnetic field goes like

$$
\Delta \Gamma\left(p, p_{\perp}, m, e B\right) \approx 1-0.003\left(\frac{e B}{m^{2}}\right)^{2} .
$$

In particular, for a magnetic field strength of $e B / \mathrm{m}^{2}=0.2$, it represents a suppression of $0.012 \%$. The corresponding dependence is so weak that it can hardly be detected in experiments; nonetheless, we present it for its theoretical interest and to show the tendency of the decay process under the influence of the magnetic field and contrast the behaviors at low and large momenta.

In the large transverse momentum regime, the magnetic field effect is more remarkable, and can be appreciated in the following figures. The behavior of the decay rate with the magnetic field, for the high momentum approximation, can be seen in Fig. 3, where the factor $g^{2} / 16 \pi$ was ignored. We plotted a log-graph, in order to remark the variation of the decay rate, which is highly affected by the Lorentz factor in this regime. Here, we can notice that the decay process follows a different behavior: the increase of the magnetic field enhances the decay width and this is magnified as the transverse momentum grows. To separate the relativistic effects on the decay width and highlight the pure magnetic field contribution, in Fig. 4 , we plot $\Delta \Gamma$ as a function of $e B / \mathrm{m}^{2}$ by using the high momentum approximation given by Eq. (23), for different values of the transverse momentum. 


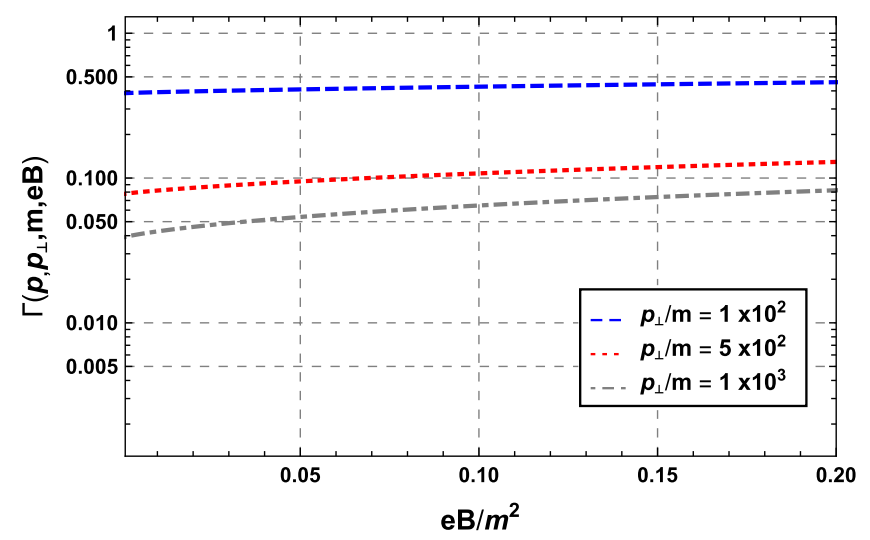

FIG. 3. Decay width (in units of energy) as a function of the magnetic field for different values of the transverse momentum, for the high momentum approximation, taking $p / m=5$.

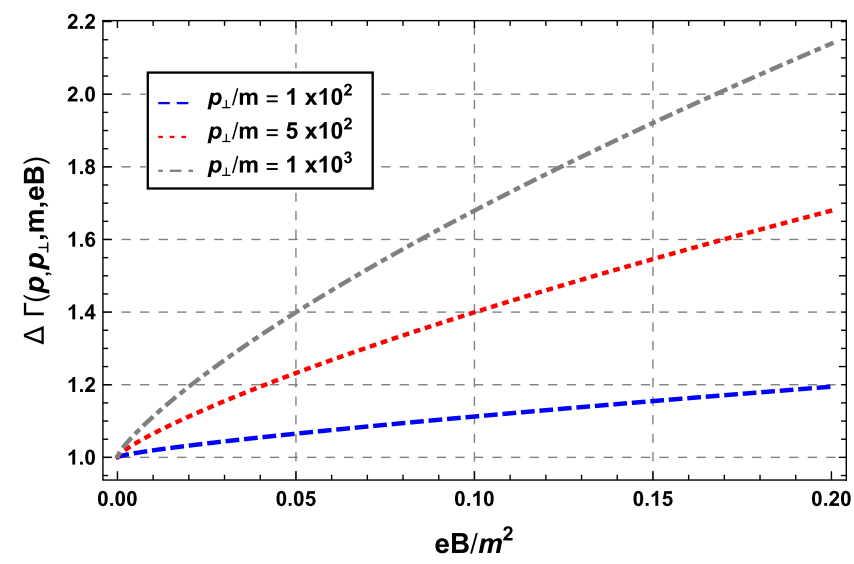

FIG. 4. Decay response to the external magnetic field as a function of the magnetic field strength, for different values of the transverse momentum, in the high momentum approximation, taking $p / m=5$.

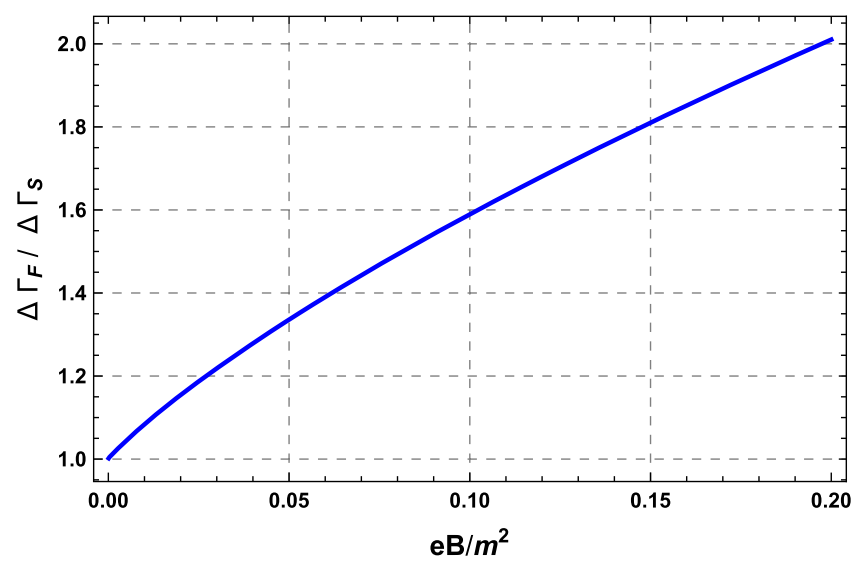

FIG. 5. Ratio of the decay responses to the external magnetic field for two different channels, $\phi \rightarrow \bar{\psi}+\psi$ and $\phi \rightarrow \varphi+\varphi$, where $\psi$ and $\varphi$ are charged fermions and scalars, respectively. We plot this ratio as a function of the magnetic field in the high momentum approximation, for $p_{\perp} / m=10^{3}$, and, as usual, we take $p / m=5$.
In order to explore the rôle played by the spin of the particle products in the decay process, in Fig. 5, we compare the $\Delta \Gamma$ for fermions, our Eq. (26) for the decay width in the high momentum approximation, with the $\Delta \Gamma$ for scalars in the same approximation, obtained in a previous work [22], for $p_{\perp} / m=10^{3}$. The behavior in Fig. 5 seems to indicate that, as the magnetic field grows, the progenitor scalar particle prefers to decay to a pair of charged fermions than to a pair of scalars, for large momenta. More work is needed, in the different kinematical regimes, in order to define the rôle of the statistics of the decay products in the presence of an external magnetic field. This work is in progress and will be published elsewhere.

\section{CONCLUSIONS}

In this work we have studied the magnetic field effects on the decay process in vacuum of a neutral scalar boson into two charged fermions. Focusing on a weak magnetic field, we carried out a perturbative approach and found that, depending on the progenitor particle kinematics, the response is inhibited or enhanced by the magnetic field. In the low transverse momentum approximation, which allows us to explore the decaying particle rest frame, we observe that the external field suppresses the decay width, meanwhile, for the high transverse momentum approximation, the effect is the opposite: as the field strength increases, the pair creation rate is enhanced.

In the low momentum approximation, we found that, for a fixed transverse momentum, the decay width $\Gamma^{B}$, as well as the response $\Delta \Gamma$, decreases as the magnetic field strength grows. This effect could be associated with the phase space reduction of the charged fermions which is caused by the increasing separation of the Landau levels with the magnetic field strength.

On the other hand, in the high momentum regime, we found that $\Gamma^{B}$ increases with the magnetic field intensity, for a fixed transverse momentum. The magnetic field effect on the decay process was emphasized by considering the ratio between the full expression (containing both the vacuum and magnetic contribution) and the pure vacuum expression (Fig. 4). In this way, the relativistic effect related to the dilatation of time was also avoided. The magnetic field rôle on this kinematical regime could have an interesting effect in the context of Higgs physics decaying into fermionic channels [54].

As we mentioned in the Introduction, many different results can be found in the literature for the effect of the magnetic field on the decay process. Since an ingredient that could be important is the spin, in order to shed some light on its rôle, we compared the response of the system to the magnetic field in two different channels: $\phi \rightarrow \bar{\psi}+\psi$ and $\phi \rightarrow \varphi+\varphi$, where $\psi$ and $\varphi$ are charged fermions and scalars, respectively. This was done, in the high momentum regime, by taking the ratio between the findings in the present work and the results in Ref. [22], and shown in Fig. 5. We observed that, in this case, the magnetic field impact is more important 
on the decay process to charged fermions than to charged scalars. These results may indicate that the spin-magnetic field interaction increases the pair production in the fermion case.

The situation studied in this work could be relevant in different physical scenarios, especially in dilepton production in heavy-ion collisions, direct URCA processes in neutron stars, or during an early stage of the Universe's evolution.

\section{ACKNOWLEDGMENTS}

J. J. U. would like to thank Efrain Ferrer and Vivian de la Incera for stimulating discussions. Support for this work has been received in part from Universidad Nacional Autónoma de México-Programa de Apoyo a Proyectos de Investigación e Innovación Tecnológica (UNAMPAPIIT) under Grants No. IN117817 and No. IA107017.

\section{APPENDIX: ALTERNATIVE WEAK FIELD APPROXIMATION IN THE LOW TRANSVERSE MOMENTUM REGIME}

In the literature, it is common to find a different weak field approximation that consists of expanding the full charged particle propagators, given by Eq. (4), in powers of $e B$, which differs from the weak field approach done in this work in Sec. III A. In order to show that these two approaches give the same result, in this Appendix we calculate the scalar decay width resorting to the first approach. With this goal in mind, let us start by performing a Taylor's expansion in powers of $e B$ up to $\mathcal{O}\left((e B)^{2}\right)$ in Eq. (4), that is

$$
\begin{aligned}
S_{F}^{B}(k) \approx & \int_{0}^{\infty} d s e^{-i s\left(m^{2}-k^{2}\right)}\left[\not k+m-i \Sigma_{3}\left(\not k_{\|}+m\right) e B s\right. \\
& \left.+\frac{1}{3}\left(i s k_{\perp}^{2}(\not k+m)+3 \not k_{\perp}\right)(e B s)^{2}\right] .
\end{aligned}
$$

Note that, in the above equation, the integration over the Schwinger's proper time becomes trivial and once this is done, the fermion propagator coincides with Eq. (48) in Ref. [55]. Nonetheless, to keep track of the logic worked out in this paper, we are going to work with the propagator in its form in Eq. (A1).

Using Eq. (A1) in Eq. (6), and after some algebraic manipulation, the scalar self-energy reads

$$
\begin{aligned}
\Sigma^{B}(p)= & 4 \int \frac{d^{4} k}{(2 \pi)^{4}} \int d s_{1} d s_{2} e^{i s_{1}\left(k^{2}-m^{2}\right)+i s_{2}\left((k-p)^{2}-m^{2}\right)} \\
& \times\left\{\left(k^{2}-k \cdot p+m^{2}\right)+e B^{2}\left[\left(k^{2}-k \cdot p+m^{2}\right)\right.\right. \\
& \times\left(i \frac{1}{3} s_{1}^{3} k_{\perp}^{2}+i \frac{1}{3} s_{2}^{3}(k-p)_{\perp}^{2}-s_{1} s_{2}\right) \\
& \left.\left.+\left(k_{\perp}^{2}-(k \cdot p)_{\perp}\right)\left(s_{1}^{2}+s_{2}^{2}+s_{1} s_{2}\right)\right]\right\},
\end{aligned}
$$

where the trace has been done.
Now, by using the change of variable in Eq. (8) and performing the Gaussian integration over the internal momenta, the above equation takes the form

$$
\begin{aligned}
\Sigma^{B}(p)= & \frac{g^{2}}{8 \pi^{2}} \int_{0}^{\infty} d s \int_{-1}^{1} d v e^{-i s\left(m^{2}-\frac{1-v^{2}}{4} p^{2}\right)}\left\{\frac{2 i}{s^{2}}+\frac{m^{2}-\frac{1-v^{2}}{4} p^{2}}{s}\right. \\
& +\frac{(e B)^{2}}{3}\left[\left(\frac{1-v^{2}}{4} p^{2}-m^{2}\right)\left(s-i s^{3}\left(\frac{1-v^{2}}{4}\right)^{2} p_{\perp}^{2}\right)\right. \\
& \left.\left.-\frac{s}{16}\left(3 v^{4}-14 v^{2}+11\right) p_{\perp}^{2}\right]\right\},
\end{aligned}
$$

where the first two terms correspond to the vacuum scalar self-energy, meanwhile the rest of the terms account for the magnetic field effect. Focussing our attention on the magnetic contribution, it is not hard to see that, once the integration over $s$ and $v$ are done, its imaginary part reads

$$
\begin{aligned}
\Im\left(\tilde{\Sigma}^{B}(p)\right)= & \frac{g^{2}}{8 \pi} \frac{4(e B)^{2}}{3 p\left(p^{2}-4 m^{2}\right)^{1 / 2}} \\
& \times\left(1+\frac{2 p_{\perp}^{2}\left(3 m^{4}-p^{2}\left(p^{2}-m^{2}\right)\right)}{p^{4}\left(p^{2}-4 m^{2}\right)}\right),
\end{aligned}
$$

which coincides with the magnetic contribution inside the square brackets in Eq. (17), once it is simplified. We also obtained this result by using the propagator in Eq. (48) of Ref. [55], following the standard procedure with Feynman parametrization.

In this way, we have checked that the order of the expansion and the integration does not affect the result, indicating that the magnetic field does not affect the selfenergy analytical structure.

Nevertheless, it is important to note that using the full propagators we can explore any kinematical region, including the low and high transverse momentum regions, meanwhile, if we use the expanded propagators, we are restricted to the low momentum region. Furthermore, note that the Schwinger's proper time procedure allows us to incorporate the whole magnetic field effect in the propagators, which cannot be done using the Feynman parameter. Note that when using the approximated propagators, the loop internal momenta are assumed to be small compared with the mass and the magnetic field, which is the main difference with our approximation, since in our approach the internal loop momenta are completely arbitrary. However, both approaches lead to the same result for the self-energy imaginary part within this kinematical regime. 
[1] V. L. Ginzburg and L. D. Landau, Zh. Eksp. Teor. Fiz. 20, 1064 (1950).[L. D. Landau, Collected Papers (Pergamon Press, Oxford, 1965), p. 546].

[2] M. Alford, K. Rajagopal, and F. Wilczek, Phys. Lett. B 422, 247 (1998).

[3] P. W. Anderson and N. Itoh, Nature (London) 256, 25 (1975); E. Flowers and N. Itoh, Astrophys. J. 230, 847 (1979).

[4] P. W. Higgs, Phys. Rev. Lett. 13, 508 (1964); G. S. Guralnik, C. R. Hagen, and T. W. B. Kibble, Phys. Rev. Lett. 13, 585 (1964).

[5] G. Aad et al. (ATLAS Collaboration), Phys. Lett. B 716, 1 (2012); S. Chatrchyan et al. (CMS Collaboration), Phys. Lett. B 716, 30 (2012).

[6] M. N. Chernodub, Phys. Rev. D 82, 085011 (2010).

[7] T. Matos, F. S. Guzmán, and L. A. Ureña-López, Classical Quantum Gravity 17, 1707 (2000).

[8] A. de la Macorra and G. Piccinelli, Phys. Rev. D 61, 123503 (2000).

[9] A. D. Linde, Particle Physics and Inflationary Cosmology (CRC Press, Boca Raton, FL, 1990); V. Mukhanov, Physical Foundations of Cosmology (Cambridge University Press, Cambridge, England, 2005).

[10] I. A. Shovkovy, Magnetic Catalysis: A Review. Strongly Interacting Matter in Magnetic Fields, edited by D. Kharzeev, K. Landsteiner, A. Schmitt, and H.-U. Yee, in Lecture Notes in Physics (Springer, Berlin, Heidelberg, 2013), Vol. 871, p. 13.

[11] F. Preis, A. Rebhan, and A. Schmitt, J. High Energy Phys. 03 (2011) 033; G. S. Bali, F. Bruckmann, G. Endrodi, F. Gruber, and A. Schaefer, J. High Energy Phys. 04 (2013) 130.

[12] R. R. Caldwell, L. Motta, and M. Kamionkowski, Phys. Rev. D 84, 123525 (2011); F. R. Urban, J. Cosmol. Astropart. Phys. 12 (2011) 012; T. Suyama and J. Yokoyama, Phys. Rev. D 86, 023512 (2012); T. Fujita and S. Mukohyama, J. Cosmol. Astropart. Phys. 10 (2012) 034; G. Piccinelli, A. Sanchez, A. Ayala, and A. J. Mizher, Phys. Rev. D 90, 083504 (2014); T. Kahniashvilia, A. Brandenburgd, R. Durrer, A. G. Tevzadze, and W. Yin, J. Cosmol. Astropart. Phys. 12 (2017) 002.

[13] M. Giovannini and M. E. Shaposhnikov, Phys. Rev. D 57, 2186 (1998); P. Elmfors, K. Enqvist, and K. Kainulainen, Phys. Lett. B 440, 269 (1998); K. Kajantie, M. Laine, J. Peisa, K. Rummukainen, and M. Shaposhnikov, Nucl. Phys. B544, 357 (1999); A. Sanchez, A. Ayala, and G. Piccinelli, Phys. Rev. D 75, 043004 (2007); A. Ayala, G. Piccinelli, and G. Pallares, Phys. Rev. D 66, 103503 (2002).

[14] S. Chakrabarty, D. Bandyopadhyay, and S. Pal, Phys. Rev. Lett. 78, 2898 (1997); D. Ebert, K. G. Klimenko, M. A. Vdovichenko, and A. S. Vshivtsev, Phys. Rev. D 61, 025005 (1999); E. J. Ferrer, V. de la Incera, and C. Manuel, Phys. Rev. Lett. 95, 152002 (2005); E. V. Gorbar, V. A. Miransky, and I. A. Shovkovy, Phys. Rev. D 83, 085003 (2011); E. J. Ferrer and V. de la Incera, Magnetism in Dense Quark Matter. Strongly Interacting Matter in Magnetic Fields, edited by D. Kharzeev, K. Landsteiner, A. Schmitt, and H.-U. Yee, in Lecture Notes in Physics (Springer, Berlin, Heidelberg, 2013), Vol. 871, p. 399.
[15] E. S. Fraga and A. J. Mizher, Phys. Rev. D 78, 025016 (2008); A. J. Mizher, M. N. Chernodub, and E. S. Fraga, Phys. Rev. D 82, 105016 (2010); A. Ayala, C. A. Dominguez, L. A. Hernandez, M. Loewe, and R. Zamora, Phys. Rev. D 92, 096011 (2015).

[16] V. A. Miransky and I. A. Shovkovy, Phys. Rep. 576, 1 (2015); J. O. Andersen, W. R. Naylor, and A. Tranberg, Rev. Mod. Phys. 88, 025001 (2016).

[17] I. Akal, S. Villalba-Chávez, and C. Müller, Phys. Rev. D 90, 113004 (2014); A. Gonoskov, I. Gonoskov, C. Harvey, A. Ilderton, A. Kim, M. Marklund, G. Mourou, and A. Sergeev, Phys. Rev. Lett. 111, 060404 (2013); S. Meuren, K. Z. Hatsagortsyan, C. H. Keitel, and A. Di Piazza, Phys. Rev. D 91, 013009 (2015).

[18] M. Kawaguchi and S. Matsuzaki, Eur. Phys. J. A 53, 68 (2017).

[19] A. Bandyopadhyay and S. Mallik, Eur. Phys. J. C 77, 771 (2017).

[20] G. G. Raffelt, Stars as Laboratories for Fundamental Physics (University of Chicago Press, Chicago, 1996).

[21] A. K. Harding and D. Lai, Rep. Prog. Phys. 69, 2631 (2006); J. M. Lattimer and M. Prakash, Phys. Rep. 442, 109 (2007); A. Y. Potekhin, Usp. Fiz. Nauk 180, 1279 (2010), Phys. Usp. 53, 1235 (2010); D. Lai, Space Sci. Rev. 191, 13 (2015).

[22] G. Piccinelli and A. Sanchez, Phys. Rev. D 96, 076014 (2017).

[23] M. Bastero-Gil, G. Piccinelli, and A. Sanchez, Astron. Nachr. 336, 805 (2015).

[24] A. Bandyopadhyay, R. L. S. Farias, and R. O. Ramos, Phys. Rev. D 98, 076007 (2018).

[25] A. V. Borisov, A. S. Vshivtsev, V. C. Zhukovskii, and P. A. Eminov, Usp. Fiz. Nauk 167, 241 (1997).

[26] W. Tsai and T. Erber, Phys. Rev. D 12, 1132 (1975); 10, 492 (1974).

[27] L. F. Urrutia, Phys. Rev. D 17, 1977 (1978).

[28] A. V. Kuznetsov, N. V. Mikheev, and L. A. Vassilevskaya, Phys. Lett. B 427, 105 (1998); A. V. Kuznetsov, N. V. Mikheev, G. G. Raffelt, and L. A. Vassilevskaya, Phys. Rev. D 73, 023001 (2006).

[29] N. V. Mikheev and N. V. Chistyakov, JETP Lett. 73, 642 (2001).

[30] A. Erdas and M. Lissia, Phys. Rev. D 67, 033001 (2003).

[31] K. Bhattacharya and S. Sahu, Eur. Phys. J. C 62, 481 (2009).

[32] P. Satunin, Phys. Rev. D 87, 105015 (2013).

[33] G. S. Bali, B. B. Brandt, G. Endrődi, and B. Gläßle, Phys. Rev. Lett. 121, 072001 (2018); EPJ Web Conf. 175, 13005 (2018).

[34] F. Karbstein, Phys. Rev. D 88, 085033 (2013).

[35] K. Sogut, H. Yanar, and A. Havare, Acta Phys. Pol. B 48, 1493 (2017).

[36] M. V. Chistyakov, A. V. Kutznetzov, and N. V. Mikheev, Phys. Lett. B 434, 67 (1998).

[37] S. Ghosh, A. Mukherjee, M. Mandal, S. Sarkar, and P. Roy, Phys. Rev. D 96, 116020 (2017).

[38] A. Ayala, C. A. Dominguez, S. Hernandez-Ortiz, L. A. Hernandez, M. Loewe, D. Manreza Paret, and R. Zamora, arXiv:1805.07344.

[39] P. Chakraborty, arXiv:1711.04404. 
[40] D. E. Kharzeev, L. D. McLerran, and H. J. Warringa, Nucl. Phys. A803, 227 (2008); V. V. Skokov, A. Yu. Illarionov, and V. D. Toneev, Int. J. Mod. Phys. A 24, 5925 (2009).

[41] A. Berera and L. Z. Fang, Phys. Rev. Lett. 74, 1912 (1995); 75, 3218 (1995); A. Berera and R. O. Ramos, Phys. Rev. D 63, 103509 (2001).

[42] M. Bastero-Gil, A. Berera, and R. O. Ramos, J. Cosmol. Astropart. Phys. 09 (2011) 033.

[43] L. M. H. Hall and I. G. Moss, Phys. Rev. D 71, 023514 (2005).

[44] T. E. Clarke, P. P. Kronberg, and H. Boehringer, Astrophys. J. 547, L111 (2001); K. Dolag, M. Kachelriess, S. Ostapchenko, and R. Tomas, Astrophys. J. Lett. 727, L4 (2011); R. Durrer and A. Neronov, Astron. Astrophys. Rev. 21, 62 (2013); E. J. Kim, P. P. Kronberg, G. Giovannini, and T. Venturi, Nature (London) 341, 720 (1989); A. Bonafede, L. Feretti, M. Murgia, F. Govoni, G. Giovannini, D. Dallacasa, K. Dolag, and G. B. Taylor, Astron. Astrophys. 513, A30 (2010); Y. Xu, P. P. Kronberg, S. Habib, and Q. W. Dufton, Astrophys. J. 637, 19 (2006).

[45] A. Neronov and A. Vovk, Science 328, 73 (2010); F. Tavecchio, G. Ghisellini, L. Foschini, G. Bonnoli, G. Ghirlanda, and P. Coppi, Mon. Not. R. Astron. Soc. 406, L70 (2010).

[46] S. Matinyan and G. Savvidy, Nucl. Phys. B134, 539 (1978); K. Enqvist and P. Olesen, Phys. Lett. B 329, 195 (1994).
[47] M. S. Turner and L. M. Widrow, Phys. Rev. D 37, 2743 (1988).

[48] P. A. R. Ade et al. Astron. Astrophys. 594, A19 (2016).

[49] J. Schwinger, Phys. Rev. 82, 664 (1951).

[50] M. E. Peskin, An Introduction to Quantum Field Theory (Westview Press, Boulder, CO, 1995); V. B. Berestetskii, L. P. Pitaevskii, and E. M. Lifshitz, Quantum Electrodynamics (Pergamon Press, New York, 1982).

[51] E. Butkov, Mathematical Physics (Addison-Wesley, Reading, MA, 1968).

[52] M. Stone and P. Goldbart, Mathematics for Physics: A Guided Tour for Graduate Students (Cambridge University Press, Cambridge, England, 2009).

[53] O. Vallée and M. Soares, Airy Functions and Applications to Physics (Imperial College Press, London, England, 2004); I. S. Gradshteyn and I. M. Ryzhik, Table of Integrals, Series, and Products, edited by A. Jeffrey and D. Zwillinger (Academic Press, New York, 2013).

[54] M. Tanabashi et al. (Particle Data Group), Phys. Rev. D 98, 030001 (2018); A. M. Sirunyan et al. (CMS Collaboration) Phys. Rev. Lett. 121, 121801 (2018); V. Khachtryan et al. (CMS Collaboration), Phys. Lett. B 744, 184 (2015).

[55] T.-K. Chyi, C.-W. Hwang, W. F. Kao, G.-L. Lin, K.-W. Ng, and J.-J. Tseng, Phys. Rev. D 62, 105014 (2000). 